\title{
OPTIMIZATION OF CLASSROOM ILLUMINATION SYSTEM BASED ON NEURAL NETWORK ALGORITHM
}

\author{
Hongwu ZENG \\ Chongqing Medical University Institute of Medical Information, Chongqing, 400016 China \\ E-mail: osrywh@163.com
}

\begin{abstract}
The optimization of classroom illumination system based on neural network algorithm was studied in order to optimize the classroom illumination system, which can effectively relieve the students " visual fatigue and promote the learning efficiency. The neural network algorithm was used. First of all, on the basis of objective evaluation of classroom illumination system, the design algorithm of university illumination based on neural network was designed creatively. Secondly, the lower-machine program of intelligent Illumination system in university was designed, and the algorithm and model of the design at last were tested. Finally, the design algorithm and the model were tested. The results show that the design can play a good role in optimizing the classroom illumination system.
\end{abstract}

Keywords: neural network algorithm, classroom lighting, optimization design

\section{INTRODUCTION}

In recent years, the economic strength of our country has been greatly improved and the degree of civilization of the society is increasing, which is inseparable from the development of education. Education has also reached an unprecedented level of attention in the whole society [1]. The size and number of education for education is also rising, especially for higher education, carrying the culture for the motherland and the task of scientific research gave birth to a man of tremendous promise, which has attracted the attention of the whole society. Col- leges and universities are not only large and more personnel, but also the distribution of personnel is more scattered [2]. Because most of the universities in our country are still taking traditional management, the waste of energy is still common. It is understood that the water and electricity expenditure of most colleges and universities in China can reach about twenty percent of the annual total expenditure of the school [3]. Especially for the consumption of electric energy, the proportion of total energy consumption is increasing, and the lighting system can make up more than fifty percent of the total energy consumption. Colleges and universities are different from primary and secondary schools, and the time and time of teaching and rest are relatively irregular [4]. And there are plenty of rooms; basically every classroom can not guarantee the people all day long. All day long in the classroom illumination in each period are different, so the lamp can not be timely closed waste of energy phenomenon, even "long light" phenomenon is still widespread there. Now most colleges and universities have adopted the way of multimedia teaching, using projectors to play slides instead of traditional teaching. Then you need to pull the curtains open lamps. The majority of colleges and universities teaching building for the first wave morning students turn on the lights, the class also no one thinks until the evening before the seal floor attendant uniform closed. Such a waste of energy not only brings a heavy burden to the running of colleges and universities, but also brings adverse effects to the development of public welfare activities of "energy saving and emission reduction" in the whole socie- 


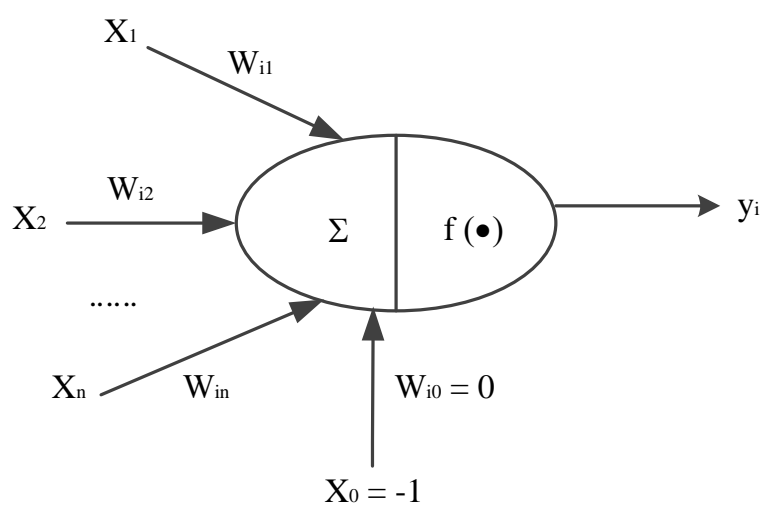

Fig.1. Neuronal structure

ty [5]. Therefore, how to effectively save energy has become a problem to be solved by the institutions of higher learning.

\section{STATE OF THE ART}

Intelligent lighting control system is an important part of intelligent building. With the continuous development of intelligent building, lighting system is no longer just for bringing light and simplicity to people, but is developing towards comfort and energy saving direction [6]. Especially for energy saving, for the important mission of intelligent lighting control system, it also takes on the task of building a saving society and promoting the sustainable development of the country. The main form of power generation at the present stage of our country is still thermal power generation. Especially in the north, if the intelligent lighting control system can be developed vigorously, the consumption of electricity can be reduced and the consumption of coal is reduced. This is of the same significance for improving the air quality and reducing the fog. In recent years, with the continuous development and mutual promotion of computer technology, automatic control technology, network communication technology and microelectronic technology, the development of intelligent lighting control system is also deepening [7]. According to the different environment, the users can make use of the intelligent lighting control system to set different requirements according to their own actual conditions. The environmental information is collected through the automatic acquisition system. The information is analyzed logically and inferred by the system, and the feedback processing signal is formed so as to achieve the best lighting control effect [8].

\section{METHODOLOGY}

\subsection{Neural Network Based Lighting Design Algorithm for Colleges and Universities}

Neural networks, also called artificial neural networks or neural computing, can be seen as the abstraction and modelling of a human brain or a biological neural network. In order to adapt to the environment and learning environment in the way of simulating biological interaction, it is an important part of artificial intelligence science and can solve all kinds of complex problems that human beings can't solve. The three main parts of the neural network are neuron, network topology and training algorithm. A neural network can be seen as a parallel processing system composed of many neurons or nodes connected by each other. The structure and function of single neuron seem simple, but the storage system formed by multiple neurons is distributed. Because of such characteristics, neural network has strong self-learning ability and high fault tolerance rate. The structure of the neuron is shown in Fig. 1.

Among them, $x_{1}-x_{n}$ represent the input signal of the neuron. $w_{i j}$ - represents the weight of the connection between the neuron $j$ and the neuron $i, \theta$ stands for the threshold value also called bias. The expression of the relationship between the input and output of the neuron $i$ is

$$
n e t_{i}=\sum_{j=1}^{n} w_{i j} x_{j}-\theta \quad y_{i}=f\left(\text { net }_{i}\right) .
$$

The output of the neuron $i$ is $y_{1}$. net is called net activation. The function $f$ is called an activation function. If the threshold value as the weight of a $w_{i 0}$ input to $x_{0}$ neurons in I, while type 1 can be simplified as

$$
n e t_{i}=\sum_{j=1}^{n} w_{i j} x_{j} \quad y_{i}=f\left(\text { net }_{i}\right) .
$$

If $X$ is defined as an input vector, $W$ is defined as a weight vector, then:

$$
X=\left[x_{0}, x_{1}, x_{2}, \ldots, x_{n}\right],
$$




$$
\omega=\left[\begin{array}{c}
\omega_{i 0} \\
\omega_{i 1} \\
\omega_{i 2} \\
\cdot \\
\cdot \\
\cdot \\
\omega_{i n}
\end{array}\right] .
$$

The output of the neuron can be transformed into the product of the vector.

$$
\begin{gathered}
\text { net }_{i}=X \omega ; \\
y_{i}=f\left(\text { net }_{i}\right)=f(X \omega) .
\end{gathered}
$$

If the net value of neurons is positive, it can be defined that neurons are in active state or excited state. If neuron net is negative, neurons can be defined as inhibitory state. Such as "threshold weighted and" neuron model is embodied in the form called M-P model (McCulloch-Pitts Model), also called the processing unit in a neural network (PE, Processing Element).

Network topology is divided into two types of feedforward and feedback networks in neural networks, including the structure of the network and the connection mode between neurons. Feedforward networks, when there is no feedback in the middle, each neuron outputs the input of the previous layer to the next layer. The feedback network refers to the existence of feedback loops between neurons. Training algorithm (also known as learning algorithm) refers to the way of adjusting weights in training process, which determines the initial weights of each connection neuron and can meet the performance of the network. The training algorithms can be roughly divided into two kinds of learning methods: supervised and unsupervised. Supervised learning can provide input mode and output mode to the network at the same time of training. And adjust weights through continuous input of different training modes so as to make output mode get closer to the expected mode. Unsupervised learning is to adjust its own parameters completely according to the input value, and the output value can accurately reflect the purpose of the characteristics of the input training sample.

\subsection{Intelligent Lighting Program Design for Colleges and Universities Based on BP Neural Network Algorithm}

The working process of the system determines the working state and the purpose of the system. Designing a correct and reasonable system workflow is also very important for the normal and stable operation of the system. The work flow chart can clearly reflect the design idea and the purpose that the designer wants to achieve for the whole system operation. The working mode and process of the system software are also determined according to the sequence of the system flow chart. This design has carried on the detailed exploration and selection to the system hardware equipment. Can we maximize the functions of this hardware, ensure the smooth operation of the hardware, and achieve the intelligent control requirements for the lighting system. The design of the work flow chart should be as accurate and reasonable as possible.

According to the related concepts of the module, the monitoring programs of the system generally include the two types of the command processing subroutine and the monitoring master program. The monitoring master can invoke a subroutine at any time. In general, when the system runs, multiple subroutines within the system can be called by the same main program at the same time. However, due to the low cost requirement of the design, the AT89S52 microcontroller may run in the running process, and the main program continuously calls the subroutine or even the dead cycle. In order to prevent the emergence of infinite circulation, we can match the main program of the slave machine into a large circular process according to a logical relationship. The functions of the lower machine in the process can be realized by calling various subroutines. In the process of designing the lower machine process, we should focus on the simple main program. The related functional modules in the system should be regarded as the subroutine under the main program. The function of the system is realized by calling the subroutine according to the design requirements. Therefore, it will become clearer and clearer to design the next machine software with this way of thinking. Even if there are any problems encountered during debugging, the causes can be found out and solved at the first time. The main program of the lower computer includes the initialization system, the related subroutine, the 


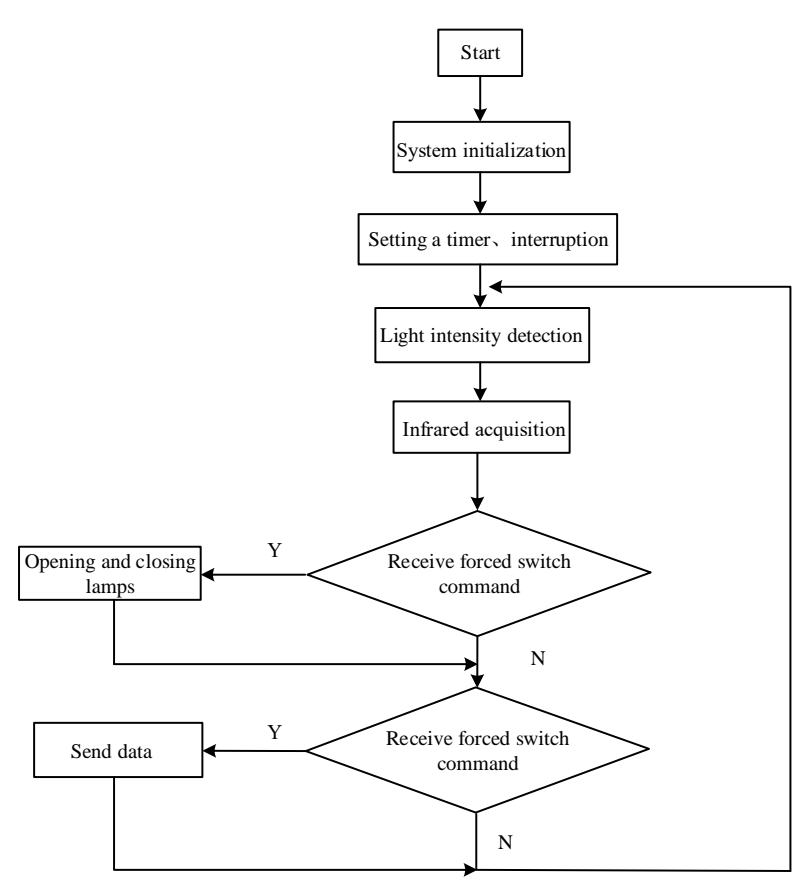

Fig.2. System work flow chart

input and output pin, the instant switch and so on. Besides the initialization and pin definition of the system, other subroutines of the system are executed repeatedly according to the workflow of the system, and each sub program will not interfere with each other. As a single chip computer program runs, it can be seen as a process to execute commands in a certain order. It is inevitable that there are some delays when running, but in fact, the speed of environment change and personnel flow in the classroom is relatively slow, and the delay of single-chip microcomputer can be ignored, which can meet the normal operation of the system. The flowchart of the main program of the lower machine is shown in Fig. 2.

The basic learning algorithm of the BP neural network is called the gradient maximum descent method. It is defined as the adjustment of weights to minimize the total error of the network, which is the gradient search technique. With expectation, the mean square error of the actual output value and the expected output value of the network is the smallest. Its network learning process can be regarded as a process of error correction and transmission coefficient correction. In the structure of BP neural network, the three layer network structure is composed of the input layer, the hidden layer and the output layer. There is no direct relationship between the hidden layer (middle layer) and the outside world, but the neuron state of the hidden layer can affect and change the input and output. The algorithm idea is the weight of network $\left(\omega_{i j}, T_{l i}\right)$ and threshold $(\theta)$ revised, so that the error along the negative gradient direction is of continuous decline, finally we obtain the satisfactory results. The learning formula of the BP neural network is as follows: among them, $\omega_{i j}$ is the network weight value between the input node and the hidden layer node. $T_{l i}$ is the network weight value between the hidden node and the output node. $t_{1}$ is the expected value of the input node. The output node is $O_{l} . W_{e}$ input in the input node $x_{j}$, the output of the middle node:

$$
y_{i}=f\left(\sum \omega_{i j} x_{j}-\theta_{i}\right) .
$$

Output node:

$$
O_{l}=f\left(\sum_{i} T_{i j}-\theta_{l}\right)
$$

Among them, the connection weight value is $\omega_{i j}$. The node domain value is $\theta_{l}$. For the output layer, the output node expects to output $t_{1}$, and the error formula is as follows:

$$
\delta_{1}=\left(t_{1}-o_{1}\right)\left(1-o_{1}\right) o_{1} .
$$

Error control:

$$
\begin{gathered}
E=\sum_{k=1}^{p} e_{k}<\epsilon, \\
e_{k}=\sum_{l=1}^{n}\left[t_{1}^{(k)}-o_{1}^{(k)}\right] .
\end{gathered}
$$

Weight correction:

$$
T_{l i}(k+1)=T_{l i}(k)+\eta \delta_{1} y_{i},
$$

where $k$ is the number of iterations. The threshold value correction:

$$
\theta_{l}(k+1)=\theta_{i}(k)+\eta \delta_{1} y_{i}
$$

Among them, $p$ is a sample number, $E$ is a sample error, and $n$ is the number of output nodes. For the correction formula of the middle layer (the input node to the intermediate node), the error formula:

$$
\delta_{i}=y_{i}\left(1-y_{i}\right) \sum_{i} \delta_{l} T_{l i}
$$

Weight correction formula: 
Table 1. Classroom Lighting Monitoring Data

\begin{tabular}{|c|c|c|c|}
\hline \multicolumn{2}{|c|}{ Input quantity } & \multicolumn{2}{c|}{ Output } \\
\hline Standard of illumination $(\boldsymbol{h})$ & Luminaire power $(\boldsymbol{W})$ & X axis coordinates $(\mathbf{c m})$ & Y axis coordinates $(\mathbf{c m})$ \\
\hline \multirow{3}{*}{150} & 40 & 171 & 225 \\
\cline { 2 - 4 } & 30 & 133 & 180 \\
\cline { 2 - 4 } & 20 & 100 & 150 \\
\cline { 2 - 4 } & 15 & 92 & 82 \\
\hline \multirow{3}{*}{200} & 40 & 200 & 150 \\
\cline { 2 - 4 } & 30 & 100 & 180 \\
\cline { 2 - 4 } & 20 & 75 & 150 \\
\cline { 2 - 4 } & 15 & 86 & 69 \\
\hline \multirow{3}{*}{300} & 40 & 171 & 129 \\
\cline { 2 - 4 } & 30 & 100 & 129 \\
\cline { 2 - 4 } & 20 & 80 & 82 \\
\hline
\end{tabular}

Table 2. The Sample Data (for Table 1)

\begin{tabular}{|c|c|c|c|c|c|c|c|c|c|c|}
\hline \multicolumn{9}{|c|}{ Input sample } & \multicolumn{2}{c|}{ Output sample } \\
\hline $\begin{array}{c}\mathrm{H} \\
(10 \mathrm{~m})\end{array}$ & $\begin{array}{c}\varphi \\
(1041 \mathrm{~m})\end{array}$ & $\begin{array}{c}\mathrm{A} \\
\left(102 \mathrm{~m}^{2}\right)\end{array}$ & $\eta$ & $\mathrm{K}$ & $P_{c}$ & $P_{f}$ & $P_{w}$ & $\begin{array}{c}E_{a v} \\
(103 \mathrm{~lx})\end{array}$ & $N(10)$ & $\begin{array}{c}E_{a v} \\
(103 \mathrm{~lx})\end{array}$ \\
\hline 0.36 & 0.3112 & 1.08 & 0.7 & 1.3 & 0.55 & 0.26 & 0.35 & 0.35 & 28 & 0.304 \\
\hline 0.36 & 0.2865 & 1.08 & 0.7 & 1.3 & 0.36 & 0.33 & 0.36 & 0.39 & 35 & 0.350 \\
\hline 0.36 & 0.2553 & 1.08 & 0.7 & 1.3 & 0.39 & 0.36 & 0.37 & 0.299 & 36 & 0.318 \\
\hline 0.36 & 0.2006 & 1.08 & 0.7 & 1.3 & 0.12 & 0.41 & 0.61 & 0.15 & 28 & 0.196 \\
\hline
\end{tabular}

$$
\omega_{i j}(k+1)=\omega_{i j}(k)+\eta \delta_{i} x_{j} .
$$

The threshold value correction:

$$
\theta_{i}(k+1)=\theta_{i}(k)+\eta \delta_{i} .
$$

\section{RESULT ANALYSIS AND DISCUSSION}

\subsection{Simulation Experiment of BP Neural Network}

In order to satisfy the standard value of illuminance, the most energy-saving lamp installation scheme is designed, which can be simulated by BP neural network. Generally in different environment, the installation position and power of the lamps and lanterns will have a certain influence on the lighting effect. The model can be built through the BP neural network. The illuminance standard and the lamp power are defined as the input quantity, the installation position of the lamp is defined as the output, and the training is carried out. The experiment selected a classroom of the teaching building as the experimental object. The input data of the BP algorithm is normalized. The input and output parameters of the network are determined, and the selection and determination of the parameters are selected according to the actual situation. The distribution of lamps and lanterns in the classroom and the measured data are shown in Table 1, and the sample data are shown in Table 2.

$P_{e}, P_{f}$, and $P_{w}$ are the characteristics of the lamp. 


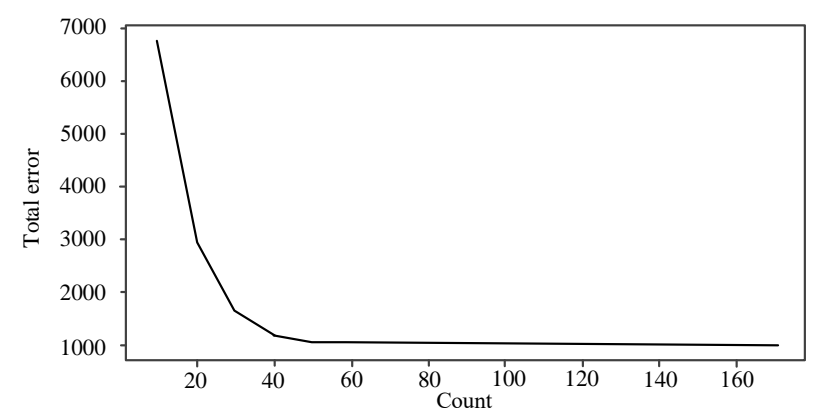

(a)

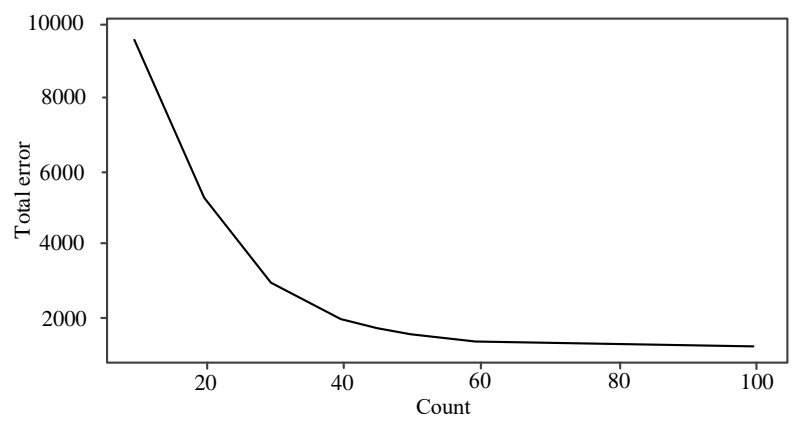

(b)

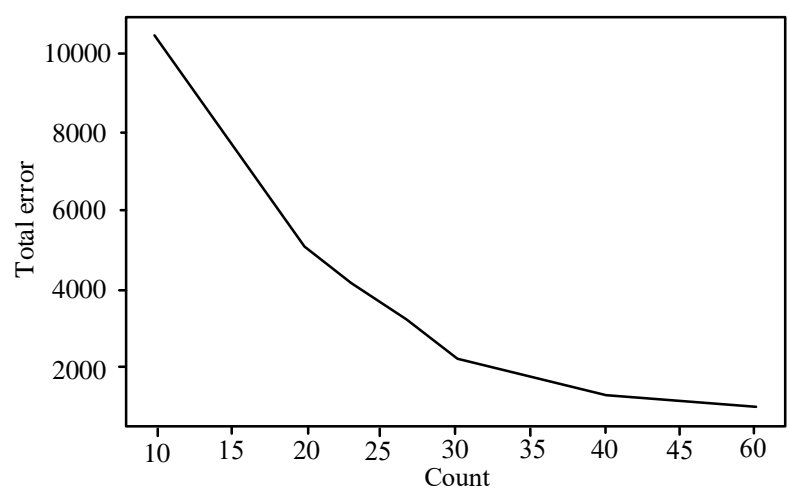

(c)

Fig.3. The BP neural network algorithm

The data used in the test show that the input amount is lighting luminance $(L C)$ and luminaire power $(W)$. The output is the minimum distance between the horizontal and vertical lights installed in a given classroom (the unit is $\mathrm{cm}$ ). The default size of the classroom is $12 \mathrm{~m} * 9 \mathrm{~m}$. For different sizes of classrooms, data can be modified by testing. The fluorescent lamp is used as the test lamp in the test. Because the 16W LED lamp in the design is basically the same with the $40 \mathrm{~W}$ fluorescent lamp on the illumination, so the simulation results of $40 \mathrm{~W}$ lamps in the test can be used as the basis for the installation location of lamps in the design. The total error of training obtained from many times is shown in Fig. 3 (a, b, c). The algorithm's error is displayed graphically, and there are shown every 10 iterations.

Then the test results are obtained. We can calculate the result of the test. Meeting the illumination requirement, if we choose $40 \mathrm{~W}$ lamps, we can install 5 lamps in the $12 \mathrm{~m} * 9 \mathrm{~m}$ classroom in the range of $X 9 \mathrm{~m}$. We can install 6 lamps in the scope of $Y$ axis, and we need to install 30 lamps in the whole classroom.

\subsection{Logical Relationship}

Generally speaking, the intelligent lighting control system can be divided into three working modes: class mode, self-study mode and mandatory mode. The logical relationship between different modes is also different. When using class mode, there are two kinds of people in the classroom. There is a light intensity in the classroom. If the indoor light intensity is enough, no matter whether the indoor lights are on or off, they should be closed. If the intensity of the indoor light is insufficient, the lamps in the classroom should be open, and the lights in the unmanned classroom should be closed. When designing binary logic relationship, it should be consistent with the output signal of the illuminance acquisition module. When the illumination intensity is enough, it is set to " 0 ", and when the illumination intensity is insufficient, it is set to " 1 ". If indoor activity is set to " 1 ", no indoor activity is set to " 0 ", indoor lighting is set to " 1 ", and indoor lighting is set to " 0 ". According to the requirements of the design, the logical relationship is formed. When using the self-study mode, a certain priority can be set for the self-study room in order to save energy. If the indoor illumination of the highest priority classroom is insufficient and the room is detected, the luminaire will be in the open state. Secondly, the first class classroom will start the intelligent control system only if the upper level lamps are turned on. The human body detection module is used to decide whether someone is indoors or not, and then decides that lamps are still open for opening. In the design of a binary logic relationship, if someone in a high priority room can be set to " 1 " room, no one is set to " 0 ". High priority indoor lamps and lanterns are set to " 1 ", and the luminaire is set to " 0 ". The next level of indoor lighting is set to " 1 ", and the lamp closes to " 0 ". According to the design requirements, a logical relationship is formed. When using coercive mode, the system will 
force the selected classroom lamps to be opened or closed to deal with all kinds of special situations. This control mode is only for simple opening and closing, and there is no logical relationship. There will be no more introductions here. In conclusion, the test results show that the design of this paper can optimize the lighting system of classroom, and classroom lighting should be optimized from lighting level, glare sensation, luminance distribution, lighting and indoor layout.

\section{CONCLUSION}

This paper took energy conservation as the starting point, takes such a large group of colleges and universities as the main research object, and took the electricity consumption in colleges and universities as the main research object. The classroom lighting system of teaching building, which is easy to cause waste, is the main research object. This paper put forward an intelligent lighting control system suitable for modern teaching buildings in colleges and universities. First of all, the algorithm and model used in this paper were described in detail. Secondly, in the overall design of the system, author learned the current development of intelligent control technology and the advantages, components, and development of intelligent lighting system by consulting related information. Based on the analysis of the existing intelligent lighting control system at home and abroad, and combined with the current situation of classroom lighting in colleges and universities in China, a classroom lighting optimization system based on neural network was constructed. In order to improve the efficiency of energy saving, the original fluorescent lamp was replaced by LED lamp. Finally, this paper simulated the best fixtures of lamps and lanterns through neu- ral network algorithm. In short, classroom lighting: the level of illumination, glare, brightness distribution should be formed by the layout optimization of lighting and interior.

\section{REFERENCES:}

1. Chen J F., Hsieh H N., Do Q H. Evaluating teaching performance based on fuzzy AHP and comprehensive evaluation approach. Applied Soft Computing, 2015. V28, pp.100-108.

2. Zhang H., Sun X. Research on low carbon supply chain performance evaluation based on AHP and fuzzy comprehensive evaluation method. Journal of Shandong University of Technology (Natural Science Edition), 2016. V1, p.16.

3. Zhaoben F., Peijie Y., Ganlin P. Empirical research on credit risk management for electric power clients. Automation of Electric Power Systems, 2005. V1, p. 17.

4. Li X., Yang S., Zhang H. Research on assessment method for power supply service quality. Power System Technology, 2004. V12, p. 8.

5. Bentaleb, Fatimazahra., Charif Mabrouki., Alami Semma. "A multi-criteria approach for risk assessment of dry port-seaport system." Supply Chain Forum: An International Journal, 2015. V16, \#4, pp.32-49.

6. Huanhuan, Wei., Lu Yuan. "Evaluation of auto dealers credit based on AHP and fuzzy comprehensive evaluation method." Modern Manufacturing Engineering, 2017. V3, p. 4.

7. Zhang N., Tao Q. Engineering Project Risk Evaluation Based on Grades Fuzzy Comprehensive Evaluation. Construction Economy, 2009. pp.S1.

8. Zhengrong, Nie. "On the Appraisal of Xincheng Tourism Resources Based on AHP-Fuzzy Comprehensive Evaluation Method." Journal of Tourism College of Zhejiang, 2016. V3, p. 6.

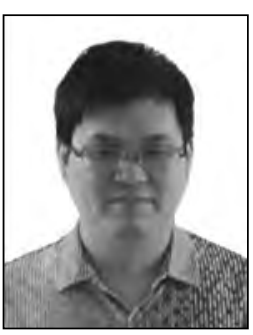

\section{Hongwu ZENG,}

lecturer of the School of Medical Information, Medical University of Chongqing. Graduated from Chongqing University in 2017 and majored in computer science. His research interests include data mining and Internet of things 